Matteo Bassetti

José-Artur Paiva

Robert G. Masterton

\section{The case for de-escalation in antimicrobial therapy: time to change our strategy in the management of septic shock?}

Received: 11 November 2013

Accepted: 16 November 2013

Published online: 6 December 2013

(C) Springer-Verlag Berlin Heidelberg and ESICM 2013

\section{Bassetti}

Infectious Diseases Division, Santa Maria Misericordia Hospital, Udine, Italy

\author{
J.-A. Paiva \\ Emergency and Intensive Care Department, Centro Hospitalar \\ Sao Joao, Porto Faculty of Medicine, University of Porto, \\ Porto, Portugal \\ R. G. Masterton (®) \\ Institute of Healthcare Associated Infection, \\ University of the West of Scotland-University Hospital \\ Crosshouse, 56A Lister Street, Kilmarnock KA2 0BB, \\ Ayrshire, UK \\ e-mail: robert.masterton@uws.ac.uk
}

The potential benefits of de-escalation during antimicrobial therapy are simple to understand and attractive in concept (Table 1) [1]. However, since the initial description of this clinical approach, reviews have remained both cautious in expressing their support and concerned about its feasibility and worthiness. In its pivotal systematic review on antimicrobial stewardship, the Infectious Diseases Society of America concluded: (1) there is good evidence to support a recommendation for its use, but (2) this conclusion is based on moderate quality evidence from well-designed clinical studies and (3) prospective randomised controlled trials are missing [2]. This conclusion was maintained in the most recent systematic review published in 2013 which found that there was no adequate, direct evidence demonstrating deescalation to be effective and safe [3]. The call was made for randomised controlled trials to answer this question. Notwithstanding the conclusions of this last systematic review, there is an increasing body of evidence in the literature based upon trials of an acceptable standard that de-escalation has a role in clinical practice.

Two reports on just such trials have recently been published in Intensive Care Medicine [4, 5]. Separately and jointly these prospective observational studies, both of which include reasonably large numbers of patients $(628$ and 101, respectively) add to the available evidence supporting de-escalation. Whereas it is now established that de-escalation is best targeted to patients with severe sepsis or septic shock, almost all studies to date have focused on medical and surgical intensive care units and cases of ventilator-associated pneumonia [1]. Both of these recent studies broaden this evidence base by examining a variety of infections including, for the first time, febrile neutropenia. It has been previously suggested that de-escalation is not an achievable goal [1]. However, regarding deescalation, clinicians are hampered by the lack of standardised definitions about the type and timing of the therapeutic modifications of this approach. Such standardized definitions are clearly needed. In previously published studies, de-escalation was achieved in 10-70\% of possible cases. These two new studies suggest that rates of 35-55\% ought to be deliverable-a very worthwhile goal. However, it remains unclear how to maximise performance in the context of defined policies versus the freedom of individual clinical practice [5]. Importantly, the authors of these two studies point out that de-escalation was only performed in approximately $50 \%$ of cases with

Table 1 The potential benefits of antimicrobial de-escalation in clinical practice

\section{Benefit}

- Unaltered clinical outcomes compared to maintenance of initial therapy

- Improved antimicrobial resistance surveillance

- Decreased antibiotic-related adverse events

- Reduced overall antimicrobial costs 
microbiologically documented cause of sepsis, pointing to frequently missed opportunities in clinical practice. Although the best opportunity for de-escalation is at around day 3 of therapy, when microbiology results are usually available, in one study only about one-third of patients were de-escalated at this stage, with the final case being achieved at day 11 [5]. More work is clearly needed to understand the dynamics of the timing of de-escalation. Faster cultural and non-cultural microbiological methods coming into clinical practice will certainly facilitate this goal, such as matrix-assisted laser desorption ionization time-of-flight mass spectrometry, technologies based on nucleic acid extraction from positive blood cultures and multiplex real-time PCR assays [6].

Although both sets of authors report that the outcome in terms of mortality rates in patients in the de-escalation cohort were at least no worse than those in patient who were not de-escalated, they also add two further insights. In the febrile neutropenia study [5], observation was extended to 1 year after discharge from the intensive care unit, which is the longest recorded follow-up period in the literature. This long follow-up did not alter the results. The results of the other study [4] actually demonstrated a protective effect from de-escalation in the group of patients who received it (odds ratio 0.54; $95 \%$ confidence interval 0.33-0.89). Although this probable beneficial effect of de-escalation has been noted before [1], there is at present no clear explanation for the phenomenon, and it remains to be determined whether this is some form of selection bias or a true effect.

In the vast majority of recent studies de-escalation has focused upon antibacterial treatment. In the febrile neutropenia study antifungal treatment was included, and it was shown that this was a part of de-escalation in $50 \%$ of cases. Although an earlier study on antifungal treatment de- escalation found that less than $40 \%$ of candidaemia patients with fluconazole-susceptible organisms were deescalated to fluconazole [7], that study, unlike the more recent febrile neutropenia study [5], did not report outcome.

It is important to recognize that many questions remain unanswered about de-escalation. There are no data yet available for antiviral treatments and very limited information on fungal infections. In general, it is still an open question as to which interventions will be effective to maximize de-escalation delivery in appropriate clinical situations. The persistently reported figures of around $50 \%$ de-escalation delivery are unacceptable and must be improved. Perhaps better microbiology laboratory-clinical unit interactions and the implementation of antimicrobial stewardship policies will be efficient strategies to avoid the vicious circle of broader initial antibiotic therapy resulting in higher levels of antimicrobial resistance [2]. One of the aspects that has not been properly explored in the recent papers is the potential microbial benefits on antimicrobial resistance rates. De-escalation and prompt discontinuation of antibiotic therapy may lessen the risk of drug-related adverse events, reduce antibiotic pressure on bacterial ecology and ultimately diminish the emergence of resistant pathogens. Also, costeffectiveness data were not addressed in either of the two cited studies. However, the two publications in this journal lend weight to questioning whether it is still reasonable not to adopt de-escalation at all because of the absence of robust randomized controlled trials. They, added to the other papers in the literature, must lead clinicians to contemplate when there will be such a weight of reasonable quality data that randomised controlled studies are no longer required to prove the point. Is that time now?

\section{Conflicts of interest None.}

\section{References}

1. Masterton RG (2011) Antibiotic deescalation. Crit Care Clin 27:149-162

2. Dellit TH, Owens RC, McGowan JE Jr, Gerding DN, Weinstein RA, Burke JP, Huskins WC, Paterson DL, Fishman NO, Carpenter CF et al (2007) Infectious Diseases Society of America and the Society for Healthcare Epidemiology of America guidelines for developing an institutional program to enhance antimicrobial stewardship. Clin Infect Dis 44:159-177

3. Silva BNG, Andriolo RB, Atallah AN, Salomão R (2013) De-escalation of antimicrobial treatment for adults with sepsis, severe sepsis or septic shock. Cochrane Database Syst Rev 3:CD007934
4. Garnacho-Montero J, GutiérrezPizarraya A, Escoresca-Ortega A, Corcia-Palomo Y, Fernández-Delgado E, Herrera-Melero I, Ortiz-Leyba C, Márquez-Vácaro JA (2013) Deescalation of empirical therapy is associated with lower mortality in patients with severe sepsis and septic shock. Intensive Care Med. doi: 10.1007/s00134-013-3077-7

5. Mokart D, Slehofer G, Lambert J, Sannini A, Chow-Chine L, Brun JP, Berger P, Duran S, Faucher M, Blache JL, Saillard C, Vey N, Leone M (2013) De-escalation of antimicrobial treatment in neutropenic patients with severe sepsis: results from an observational study. Intensive Care Med. doi: 10.1007/s00134-013-3148-9
6. Leggieri N, Rida A, François P, Schrenzel J (2010) Molecular diagnosis of bloodstream infections: planning to (physically) reach the bedside. Curr Opin Infect Dis 23:311-319

7. Shah DN, Yau R, Weston J, Lasco TM, Salazar M, Palmer HR, Garey KW (2011) Evaluation of antifungal therapy in patients with candidaemia based on susceptibility testing results: implications for antimicrobial stewardship programmes. J Antimicrob Chemother 66:2146-2151 